\title{
Smartphone-based biosensing platform evolution: implementation of electrochemical analysis capabilities
}

Patou, François; Dimaki, Maria; Svendsen, Winnie Edith; Kjærgaard, Claus; Madsen, Jan

\section{Published in:}

Proceedings of the 10th International Symposium on Medical Information and Communication Technology (ISMICT)

Link to article, DOI:

10.1109/ISMICT.2016.7498881

Publication date:

2016

Document Version

Peer reviewed version

Link back to DTU Orbit

Citation (APA):

Patou, F., Dimaki, M., Svendsen, W. E., Kjærgaard, C., \& Madsen, J. (2016). Smartphone-based biosensing platform evolution: implementation of electrochemical analysis capabilities. In Proceedings of the 10th International Symposium on Medical Information and Communication Technology (ISMICT) IEEE. https://doi.org/10.1109/ISMICT.2016.7498881

\section{General rights}

Copyright and moral rights for the publications made accessible in the public portal are retained by the authors and/or other copyright owners and it is a condition of accessing publications that users recognise and abide by the legal requirements associated with these rights.

- Users may download and print one copy of any publication from the public portal for the purpose of private study or research.

- You may not further distribute the material or use it for any profit-making activity or commercial gain

- You may freely distribute the URL identifying the publication in the public portal 


\section{Smartphone-based biosensing platform evolution: implementation of electrochemical analysis capabilities.}

\author{
François Patou, \\ Maria Dimaki, Winnie E. Svendsen \\ dept. of Micro- and Nanotechology \\ Tech. Univ. of Denmark, DTU \\ Lyngby, Denmark \\ email:frpato@nanotech.dtu.dk
}

\author{
Claus Kjægaard \\ dept. of Electrical Engineering \\ Tech. Univ. of Denmark, DTU \\ Lyngby, Denmark \\ email: clkj@dtu.dk
}

\author{
Jan Madsen \\ dept. of Applied Mathematics \\ and Computer Science \\ Tech. Univ. of Denmark, DTU \\ Lyngby, Denmark \\ email:jama@dtu.dk
}

\begin{abstract}
Lab-on-Chip technologies offer great opportunities for the democratization of in-vitro medical diagnostics to the consumer-market. Despite the limitations set by the strict instrumentation and control requirements of certain families of these devices, new solutions are emerging. Smartphones now routinely demonstrate their potential as an interface of choice for operating complex, instrumented Lab-on-Chips. The sporadic nature of home-based in-vitro medical diagnostics testing calls for the development of systems capable of evolving with new applications or new technologies for Lab-on-Chip devices. We present in this work how we evolved the first generation of a smartphone/Lab-on-Chip platform designed for evolvability. We demonstrate how reengineering efforts can be confined to the mobile-software layer and illustrate some of the benefits of building evolvable systems. We implement electrochemical capabilities on our platform prototype and carry out cyclic voltammetry to measure dopamine concentrations over several orders of magnitude.
\end{abstract}

Keywords-Lab-on-Chip; Smartphone; Point-of-Care; InVitro Medical Diagnostics; System Evolvability; PlatformBased Design;

\section{INTRODUCTION}

Recent advances in Lab-on-Chip (LoC) technologies have raised hope in the ability to better diagnose and monitor disease at the Point-of-Care (PoC). The wide panel of analytical functionalities and ever-increasing performances of LoCs have made them critical vectors for the democratization of In-Vitro Medical Diagnostics (IVMD) in the consumer-market. A certain number of limitations have so far prevented the large-scale development and adoption of direct-to-consumer LoC-based technologies. One is the increasing need for instrumentation and control: as LoCs capabilities increase, so is the complexity of their operation (e.g. optical readout instrumentation, control of digital microfluidics biochips [1], [2], etc.). Smartphonebased systems have a transformative potential in that respect [3]. Many examples already illustrate possible benefits of interfacing LoCs to smartphones, leveraging the computational power, peripherals and usability of the latter to avoid the need for developing complex instruments or controllers [3]. Nevertheless, as we argued in a recent publication, the predictable sporadic nature of home-based IVMD testing questions the justifiability of developing application-specific or non-evolvable systems [4]. So does the fast-pace evolution of the technologies underlying LoC systems (e.g. biosensing, microfluidics). We therefore advocate design for evolvability in smartphone/LoC systems and recently introduced the first generation of a platform implemented in that perspective [4]. We follow up on these initial results and present in this work how we evolved our initial design to allow the execution of electrochemical measurements. We demonstrate the operation of the latter functionality by carrying out the detection of dopamine (DA) using cyclic voltammetry (CV).

\section{MATERIALS AND METHODS}

\section{A. Platform evolution by functional composition}

We relied on a Platform-Based Design (PBD) methodology to develop our initial concept [5] and worked under the guidance of engineering design principles for changeability on which we further elaborate in this paper [6], [7]. PBD enables the mapping of specified system functions onto a target architecture [5]. This materializes in our case with the possibility to specify LoC operations (i.e. functions) at the high-abstraction mobile-software layer, for varied LoC architectures [4]. An iOS Application Programming Interface (API) gives the LoC application designer access to a set of customizable tasks (figure 1). Among them, we call elementary tasks the physical or computational functions realized at the Hardware Accessory (HWA) or LoC level. These tasks were specified according to the ideality/simplicity principle: in an attempt to maintain low system complexity while maximizing functionality [6]: we specified the original functional requirements for our platform so as to allow the recovery of low- AC-currents, such as those exhibited by siliconnanowire field effect transistor biosensors [8], [9]. Among potential means to offer this capability, we decided to rely on the lock-in amplification technique [4], [10]. We then derived the set of elementary tasks satisfying the aforementioned ideality principle. These are illustrated in figure 2a. Their definition is critical, as it requires the careful evaluation 


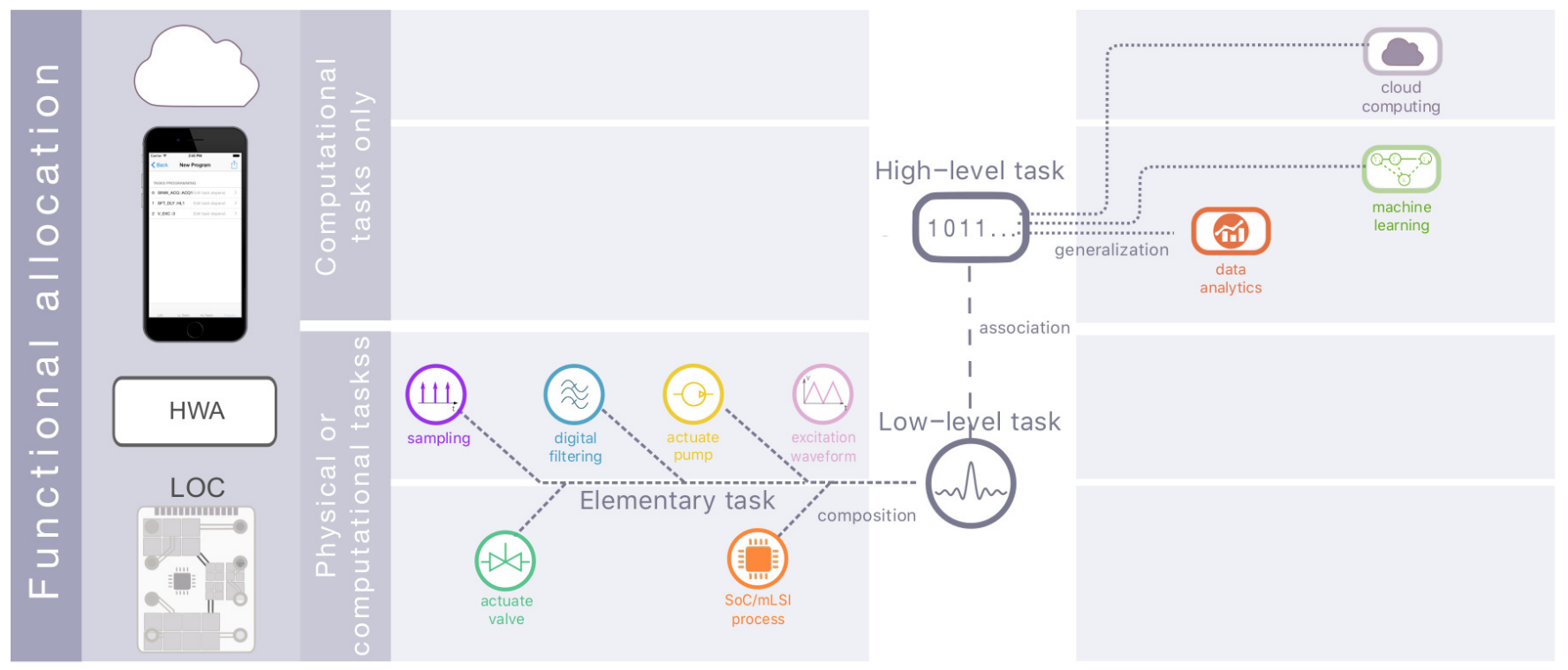

Figure 1. Platform functionality abstractions. Elementary tasks are defined as physical or computational functions performed at the low-abstraction HWA or LoC level. They can be composed at the mobile-software layer to form Low-level tasks. These low-level tasks can be associated to high-level tasks, defined as computational-only, mobile-software level or cloud-computing tasks. This abstraction and ontology enables the non-hierarchical manipulation of low- and high-level tasks at the mobile-software API level, facilitating the specification of new LoC operations

of complexity/flexibility tradeoffs: When low abstraction levels provide great flexibility, implementation complexity may be burdening (e.g. mobile-software control of every HWA CPU register), whereas high abstraction levels will limit changeability but promote simplicity.

These elementary tasks were also defined in search of modularity: modular architectures should support the reuse of modular elements or aggregation of modules and their interfaces. This property permits the modular composition of elementary tasks at the high-abstraction mobile-software layer. We refer to these compositions as low-level tasks. The composition of the lock-in amplification acquisition function is presented in figure $2 \mathrm{a}$.

Our elementary tasks are also independent: they should alone provide useful function (e.g. voltage waveform generation for LoC applications requiring specific electric fields patterns).

Finally they are integrated non-hierarchically with high-level tasks i.e. the computational-only functions associated with LoC operation and realized in mobile-software or in the cloud: the API provides a way to associate elementary tasks to high-level tasks at the same abstraction layer, allowing for instance for cloud-computing queries to be leveraged at run-time to define succeeding LoC operations.

Our prototype implementation and API rely on a HWA enabling the interfacing of an iPhone 6 (Apple Inc. CA, USA) to passive LoCs embedding current-based biosensors [4]. We built upon available elementary tasks to foster electrochemical capabilities to our platform: We imple- mented the software blocks enabling the execution of cyclic voltammetry from the composition of a voltage waveform generation and current acquisition.

\section{B. Electrochemical measurements}

Following implementation, we made use of the available $\mathrm{CV}$ acquisition capabilities and proceeded to the electrochemical quantitation of dopamine (DA) solutions obtained from dopamine hydrochloride (Sigma-Aldrich, MO, USA). A stock solution at $0.1 \mathrm{~mol} / \mathrm{L}$ and successive dilutions were prepared to obtain DA concentrations down to $5.9 \mathrm{nmol} / \mathrm{L}$. We used distilled water (Millipore Milli-Q, Bedford, MA, USA) as a solvent. CV was performed using a scan rate of $100 \mathrm{mV} / \mathrm{s}$ for potentials ranging from $\mathrm{E}=-1 \mathrm{~V}$ to $\mathrm{E}=1 \mathrm{~V}$. Current sensitivities (i.e. reciprocal of the front-end current amplifier gain) were specified programmatically (via the API) so as to maximize the signal-to-noise ratio for a given DA dilution. Each calibration point was obtained by averaging results from three measurements, obtained using a new electrode each time (screen-printed gold-electrodes C223AT, DropSens, Spain) .

\section{RESUlTS}

\section{A. Implementation of cyclic-voltammetry analysis functions}

The functional decomposition of $\mathrm{CV}$ acquisitions is provided in table 1 . The design variables over which the application designer should have control could be mapped to the settings of the available elementary tasks. CV acquisitions were thus implemented by the composition of a triangular voltage waveform generation with a synchronous current 


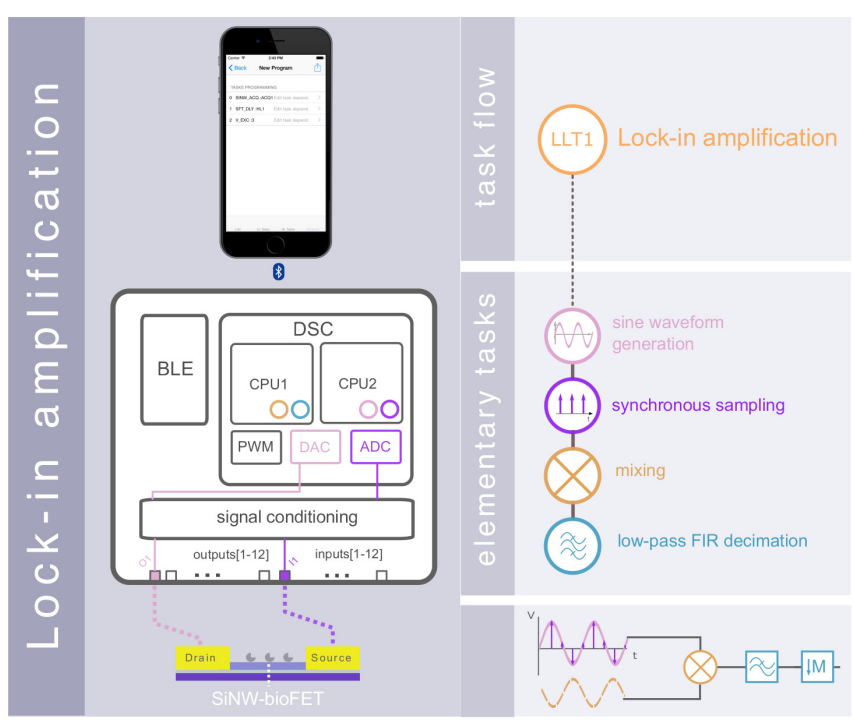

(a)

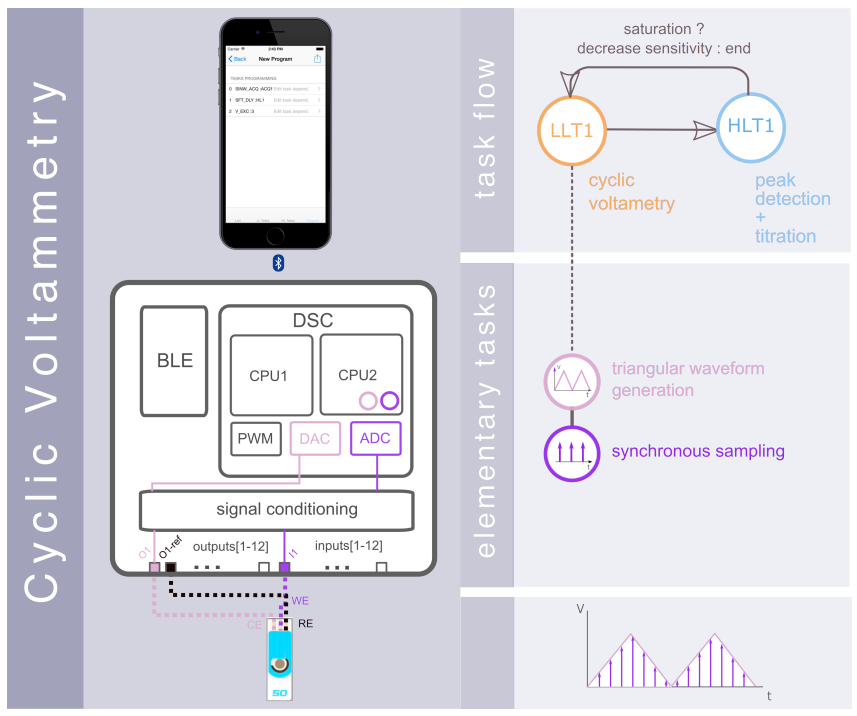

(b)

Figure 2. a - Lock in amplification function performed for the recovery of the impedance magnitude and phase from a high-impedance Silicon Nanowire (SiNW) sensor [4]. Lock-in amplification is carried out by applying a sinusoidal voltage waveform between the source and drain of the sensor, amplifying the resulting current running between source and drain and perform phase sensitive detection. (PSD). PSD itself is composed of a mixing step using the excitation reference signal and of a low-pass filtering step, both function been made available in embedded software $\mathrm{b}$ - LoC program execution diagram for $\mathrm{CV}$ acquisitions. CVs executed successively at decreasing sensitivities if current saturation is detected within the potential range where calibration is carried out. This conditional branching is realized at the mobile-software layer. Cyclic voltammetry LLT1 is composed of two synchronous elementary tasks: a triangular waveform generation and synchronous sampling. CV design variables must therefore translate into settings for the required elementary tasks.

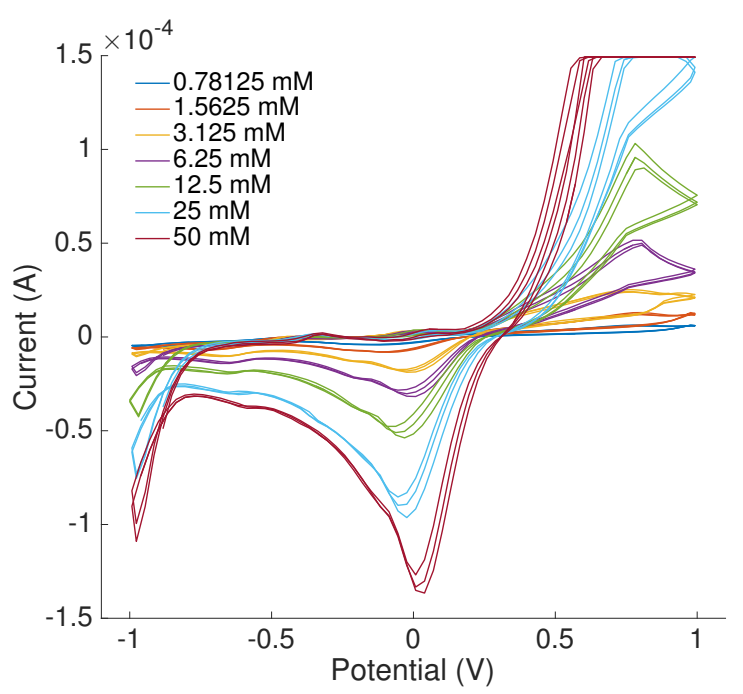

Figure 3. Mobile-phone acquisition data: Cyclic voltammograms. Saturated acquisitions are only discarded when saturation occurs within the potential range of interest.

acquisition. Both these functions could then be mapped to one of the two available hardware potentiostatic channels (figure 2b). A peak-detection algorithm (i.e. high-level task) was inserted in the LoC operation program: starting at highsensitivity, $\mathrm{CV}$ acquisitions are retrieved in $\mathrm{iOS}$ and probed for saturation. If a saturation is detected, the $\mathrm{CV}$ is repeated

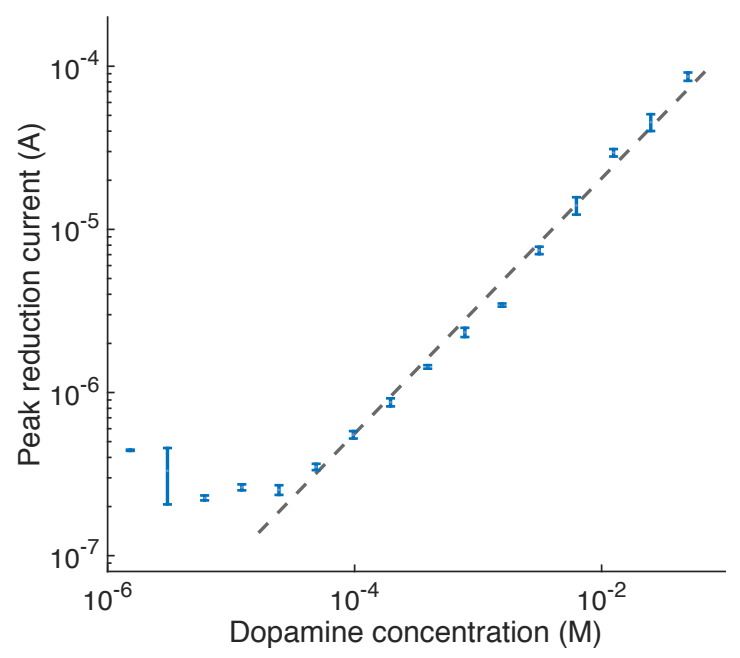

Figure 4. DA calibration curve. Calibration points are obtained from retrieving local minima around $\mathrm{E}=0 \mathrm{~V}$;

with a decreased amplifier sensitivity so as to be able to recover the reduction peak current signal (figure $2 \mathrm{~b}$ ).

\section{B. Electrochemical detection of dopamine}

After having validated the correct execution of CV acquisitions, DA quantitation was carried out. An illustration of the acquisition data retrieved at the mobile-software layer is given in figure 3. For each acquisition, a calibration point was retrieved from the identification of the local current 
Table I

FUNCTIONAL COMPOSITION OF CYCLIC VOLTAMMETRY (CV). THE ACQUISITION SETTINGS I.E. DESIGN VARIABLES FOR CV, CAN BE MAPPED TO THE DIFFERENT PARAMETERS AVAILABLE THROUGH THE elementary tasks OFFERED BY THE PLATFORM.

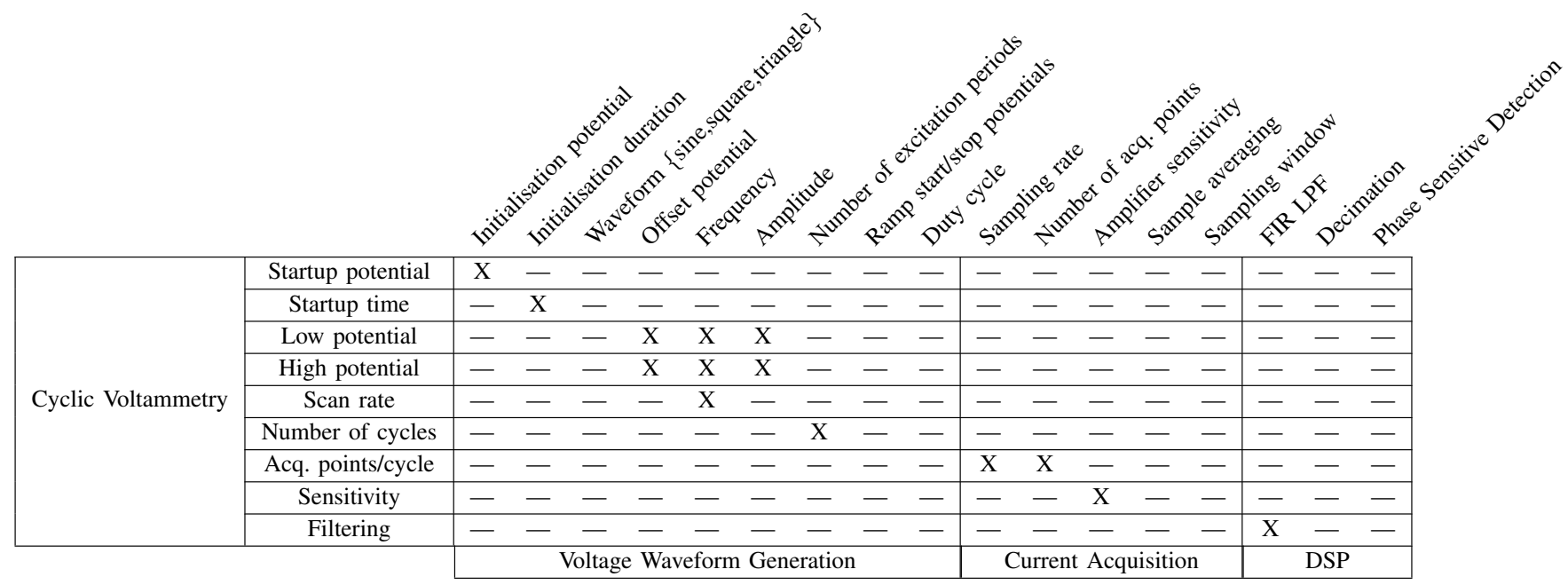

minimum at DA's reduction potential. From these points we could derive the calibration curve presented in figure $4 \mathrm{~b}$. Our calibration curve spans over several orders of magnitude of DA concentrations with a solid regression coefficients of 0.996 , benefiting from the scalability of the input current amplifiers combined with a smart algorithm implemented at the iOS software layer.

\section{DISCUSSION}

The methodology and results presented in this work illustrate the benefits of PBD coupled with the adoption of appropriate engineering design principles for the implementation of evolvable systems. The development of electrochemical capabilities for our platform required the sole functional composition of existing elementary tasks and did not require change to any other system components. This was made possible mainly because of the careful consideration of functional modularity. The experimental results provided in section III-B demonstrate the operability of the evolved platform and illustrate the potential benefit of the non-hierarchical integration of high-level analytics (here a peak-detection algorithm) with LoC operations (here $\mathrm{CV}$ acquisitions).

This specific evolution towards electrochemical instrumentation represents of course a special case: The development of other physical functions (e.g. instrumentation at the HWA level or microfluidic actuation at the LoC level) may require more drastic system changes. In such a case, the reengineering effort and cost will depend upon how change propagates throughout the entire system architecture. We are currently addressing this matter using Change Propagation Analysis (CPA) [11].

\section{CONCLUSION}

We demonstrated in this work how PBD and design for evolvability can result in low re-engineering efforts for the adoption of new LoC architectures or operations. More specifically we demonstrated how to develop electrochemical capabilities for our system by the composition of existing functional block available at the API level. Our endeavour acknowledges that change is the predominant paradigm in engineering complex systems. It finally provides evidence as to the potential benefits of design for evolvability for directto-consumer PoC systems.

\section{ACKNOWLEDGMENT}

This project is a part of the EU Marie Curie Initial Training Networks (ITN) Biomedical engineering for cancer and brain disease diagnosis and therapy development: EngCaBra. Project no. PITN-GA-2010-264417.

\section{REFERENCES}

[1] M. J. Jebrail, M. S. Bartsch, and K. D. Patel, "Digital microfluidics: a versatile tool for applications in chemistry, biology and medicine," Lab on a Chip, vol. 12, no. 14, p. 2452, 2012. [Online]. Available: http://xlink.rsc.org/?DOI= c2lc40318h

[2] P. Pop, M. Alistar, E. Stuart, and J. Madsen, Fault-Tolerant Digital Microfuidic Biochips. Springer US, 2015.

[3] B. X. Xu, A. Akay, H. Wei, S. Wang, B. Pingguan-murphy, X. Li, W. Lee, J. Hu, L. Wang, and F. Xu, "Advances in Smartphone-Based Point-of-Care Diagnostics," vol. 103, no. 2, 2015.

[4] F. Patou, M. Dimaki, W. E. Svendsen, K. Kjaegaard, and J. Madsen, "A Smart Mobile Lab-on-Chip-Based Medical Diagnostics System Architecture Designed for Evolvability," 2015 Euromicro Conference on Digital System Design, pp. 390-398, 2015. [Online]. Available: http://ieeexplore.ieee. org/lpdocs/epic03/wrapper.htm?arnumber=7302301 
[5] A. Sangiovanni-vincentelli, "Platform-Based Design and Software Design Methodology for Embedded Systems," no. December, pp. 23-33, 2001.

[6] E. Fricke and A. P. Schulz, "Design for changeability (DfC): Principles to enable changes in systems throughout their entire lifecycle," Systems Engineering, vol. 8, no. 4, pp. 342359, 2005.

[7] R. Steiner, "System Architectures and Evolvability: Definitions and Perspective," Systems Engineering, p. 5, 1998.

[8] G. Zheng, F. Patolsky, and C. Lieber, "Nanowire biosensors: a tool for medicine and life science," Nanomedicine: Nanotechnology, Biology and Medicine, vol. 2, no. 4, p. 277, 2006. [Online]. Available: http://dx.doi.org/10.1016/j. nano.2006.10.050

[9] D. Kwasny, M. Dimaki, K. B. Andersen, A. Zulfiqar, Z. Tümer, and W. E. Svendsen, "Nanoscaled Biological Gated Field Effect Transistors for Cytogenetic Analysis," in Proceedings of the 9th IEEE International Conference on Nano/Micro Engineered and Molecular Systems, 2014, pp. $130-134$

[10] N. Li, W. Wang, H. Xu, H. Yu, J. Diao, and D. D.-U. Li, "Wide-Bandwidth Biological Impedance Spectroscopy System Based on the Digital Lock-In Technique," Spectroscopy Letters, vol. 46, no. 7, pp. 476-482, 2013. [Online]. Available: http://www.tandfonline.com/doi/abs/10. 1080/00387010.2012.705801

[11] N. Ahmad, D. C. Wynn, and P. J. Clarkson, "Change impact on a product and its redesign process: A tool for knowledge capture and reuse," Research in Engineering Design, vol. 24, no. 3, pp. 219-244, 2013. 